\title{
A General Theory of Electrochemistry
}

\author{
Bruno Nilsson
}

January 15, 2022

\begin{abstract}
This paper presents a new electrochemical theory that provides an accurate description of the double-layer at the microscopic level of the electrode/electrolyte interface, which, in turn, can be used to derive and predict static and dynamic macroscopic level properties for the half-cell.

To illustrate the theory's general validity, we derive the classic Butler/Volmer and Nernst's Equations.

As a dynamic example, we will show the derivation of the electrode voltage variation with time from when an electrode contacts an inert electrolyte and compare it with actual measurements.

Another dynamic example is the derivation of the electrode voltage variation from initial contact with an electrolyte containing electrode ions until established equilibrium, ending up agreeing with Nernst's Equation.

The theory can also predict the electrode voltage response to external time-varying currents, such as described by the complex impedance of the charge transfer reaction. We will compare theory with actual impedance measurements on a $\mathrm{Cu} / \mathrm{CuSO}_{4}$ half-cell.
\end{abstract}

\section{Introduction}

The electrode/electrolyte interface reaction is of central importance in electrochemistry.

The first attempt to provide a model for the interface is due to work by (von Helmholtz, 1879) and Perrin. Their model resembled a parallel-plate capacitor with one plate in the electrode and the other in the electrolyte - hence the generic term double layer was established, with a charged layer on each side of the virtual interface. The Helmholtz-Perrin model was though too simplistic to describe basic observations at the macroscopic level adequately.

Efforts by (Gouy, 1903) and (Chapman, 1913) resulted in a significantly refined model whereby ions in the electrolyte were allowed to distribute themselves following Boltzmann's distribution. Despite these improvements, it still had severe shortcomings.

A further refined model, proposed by (Stern, 1924) was essentially a combination of the two previous models, with a Helmholtz layer next to the electrode surface combined with an outer Gouy-Chapman type of diffuse layer. Although 
this model overcame some of the shortcomings of previous models, it still provided only a conceptual, qualitative description of the double-layer. The same can also be said about more recent model proposals.

(Bockris and Reddy, 1970) provided a detailed review of the double-layer models mentioned above, including later made proposals with minor refinements. Specifically, they concluded about the Gouy-Chapman model: "The model seems to be in sharp discord with the facts. Looked at as an attempt to represent all the essential aspects of an electrified interface, the Gouy-Chapman theory might best be described as a brilliant failure. However, it represents an important contribution to a truer description of the double-layer. A more consistent theory of the double-layer is clearly needed."

The distribution of ions, the electric field, and electrode current at the microscopic level represented by the double-layer is the underlying origin of the properties and phenomena we observe at the macroscopic level of the half-cell.

This paper presents a new electrochemical theory that provides an accurate description of the double-layer at the microscopic level that, in turn, can be used to derive and predict macroscopic level properties described by, for instance, the Nernst and Butler-Volmer equations. The presumption is that the proper prediction of already known properties at the macroscopic level confirms the validity of the underlying microscopic double-layer model.

The theory's core is a partial differential equation that describes the electric field distribution near the electrode surface, based on fundamental natural constants and specific boundary conditions. The field represents, we believe, a much more accurate diffuse distribution of ions compared to the Gouy-Chapman model.

We derived the underlying basic equation in an early attempt to explain the phenomenon of Induced Polarization (Nilsson, 1970). Fundamentally this is an electrochemical cell's voltage response to a time-dependent current.

Mining companies effectively employ this phenomenon in the large-scale search for mineral deposits. The individual mineral grains in the ground, surrounded by groundwater in grain boundaries, constitute tiny electrochemical cells.

Injecting a low-frequency sine-wave current into the ground via current electrodes separated hundreds of meters apart creates an electric field that can be measured via a pair of potential electrodes separated by, let us say, 20 meters. Disseminated mineralization occurrences can be detected via the phase lag relative to the inducing current due to the Induced Polarization effect.

We realized early on that the basic equation probably provided a more general description of the electrochemical interface reaction than what we required for the explanation of the Induced Polarization effect, (Nilsson, 1973; Nilsson, 1975).

With this paper, we attempt to illustrate the theory's general nature and present it to the electrochemical community.

Section 2 presents the theory.

Section 3 deals with static phenomena. The theory's general validity is demonstrated by deriving the well-known Butler/Volmer and Nernst Equations. 
Section 4 shows the theory's ability to handle dynamic phenomena.

In Section 4.1, as an example, we derive the electrode voltage variation with time from the moment an electrode contacts an inert electrolyte. To validate the accuracy of the theoretical result, we compared it with actual voltage measurements on a zinc electrode dipped into an aqueous sodium sulfate solution.

Section 4.2 derives the electrode voltage variation from when the electrode contacts an electrolyte containing electrode ions until established equilibrium. We demonstrate that the electrode voltage will approach and eventually reach equilibrium condition, as expected, in agreement with the predictions of Nernst's Equation.

Another example of dynamic behavior is the electrode voltage response to external time-varying electrode currents. In Section 4.3, as an example, the derivation of the complex charge transfer impedance is carried out, which details the electrode voltage response to sine-wave currents.

Finally, in Section 4.3.1, we will compare these theoretical results with actual impedance measurements on a $\mathrm{Cu} / \mathrm{CuSO}_{4}$ half-cell.

Section 5 discusses how to access the various Python computer programs we developed as part of this project. Many of these are part of a Python Notebook, we have made publicly available and executable. Section 5 provides information on how to access this Notebook and run the individual programs interactively via the Internet.

\section{The Theory}

We will, in this section, formulate a theoretical model for the electrode/electrolyte interface that accurately describes the electric field in the electrolyte, as a consequence of the associated charge transfer reaction.

In the form of a partial differential equation, the model will describe the electric field both under static and dynamic conditions.

The valence electrons in a metal electrode are not bound to particular atoms but are free to move between atoms. This is why metals, in general, have a high electrical conductivity.

Water is an excellent solvent for various chemical substances like metal salts. To a very minor extent, it is also a solvent for many metals.

When a metal electrode makes contact with water, the strongly polar water molecules tend to weaken the electric forces that hold the surface atoms bound to the electrode, causing some of them to detach without their valence electrons. The detached atoms are thus free to move into the water as ions, while the electrons remain members of the electrode's cloud of free electrons.

Thus, the electrode will become negatively charged while the water receives a positive excess charge due to the metal ions. This anodic charge displacement creates, in turn, an electric field as a consequence.

If the bulk of the water already contains electrode ions, there is also a certain probability of the opposite reaction occurring, whereby electrode ions in the water recombine with electrons on the electrode surface to form electrode metal 
atoms. This cathodic charge displacement in the opposite direction creates an opposing electric field.

We will thus end up with current flows going in opposite directions. Eventually, equilibrium will occur. The two opposing current flows will be equal, and there is no further net flow of ions across the electrode/electrolyte interface. The electrode potential is now stable.

We will now study a simple single-step electrochemical reaction like:

$$
M e \rightleftharpoons M e^{z+}+z e^{-}
$$

where $M e$ is the electrode metal atom, $z$ the valence, and $e$ the elementary charge of the electron.

If we consider a small volume element containing a specific density $\rho$ of metal ions, in the the electrolyte near the electrode, it will be subject to both thermal and electric forces.

Following equation describes the thermal forces $\overrightarrow{\mathbf{F}_{T}}$ :

$$
\overrightarrow{\mathbf{F}_{T}}=-k T \nabla \rho
$$

where $k$ is Boltzmann's constant and $T$ the absolute temperature.

Likewise, the force $\overrightarrow{\mathbf{F}_{E}}$ caused by the electrical field $\overrightarrow{\mathbf{E}}$ is described by:

$$
\overrightarrow{\mathbf{F}_{E}}=z e \rho \overrightarrow{\mathbf{E}}
$$

The sum of these forces will create an ionic flow proportional to the total force acting on the volume element, as shown below:

$$
\rho \overrightarrow{\mathbf{v}}=\operatorname{const}(-k T \nabla \rho+z e \rho \overrightarrow{\mathbf{E}})
$$

or, reformulated as:

$$
\rho \overrightarrow{\mathbf{v}}=\text { const } \cdot k T\left(-\nabla \rho+\frac{z e}{k T} \rho \overrightarrow{\mathbf{E}}\right)
$$

By inspection, we realize that const. $k T$ is noting but the well-known diffusion coefficient $D$, (and consequently const is the mobility coefficient $\mu$ ). Thus we have:

$$
\rho \overrightarrow{\mathbf{v}}=D\left(-\nabla \rho+\frac{z e}{k T} \rho \overrightarrow{\mathbf{E}}\right)
$$

We will now introduce the continuity equation:

$$
\frac{\partial \rho}{\partial t}=-\nabla \cdot(\rho \overrightarrow{\mathbf{v}})
$$

and Gauss's Law:

$$
\nabla \cdot(\epsilon \overrightarrow{\mathbf{E}})=z e \rho
$$

where $\epsilon$ is the dielectric constant. 
Typically, the electrode potential is independent of the electrode's shape for practical electrode dimensions. The electrochemical reaction thus occurs close to the electrode surface. A one-dimensional Cartesian coordinate system is consequentially a suitable framework for theoretical analysis.

Equation 6 and 7 now results in:

$$
\frac{\partial \rho}{\partial t}=D \frac{\partial}{\partial x}\left(\frac{\partial \rho}{\partial x}-\frac{z e}{k T} \rho E\right)
$$

and with 8 we obtain:

$$
\frac{\partial}{\partial t}\left(\frac{\partial E}{\partial x}\right)=D \frac{\partial}{\partial x}\left(\frac{\partial^{2} E}{\partial x^{2}}-\frac{z e}{k T} E \frac{\partial E}{\partial x}\right)
$$

We expect the electric field $E(x, t)$ to be a smooth function over the entire valid range $0<\mathrm{x}$ and $0<\mathrm{t}$. This, in turn, means that $E(x, t)$ 's partial derivatives are continuous for $0<\mathrm{x}$ and $0<\mathrm{t}$. The partial differentiations of $E(x, t)$ are thus commutative. According to Schwarz's Theorem, therefore, following equation is equally valid:

$$
\frac{\partial}{\partial x}\left(\frac{\partial E}{\partial t}\right)=D \frac{\partial}{\partial x}\left(\frac{\partial^{2} E}{\partial x^{2}}-\frac{z e}{k T} E \frac{\partial E}{\partial x}\right)
$$

which can now immediately be simplified to:

$$
\frac{\partial E}{\partial t}=D\left(\frac{\partial^{2} E}{\partial x^{2}}-\frac{z e}{k T} E \frac{\partial E}{\partial x}\right)
$$

We recognize the equation as a non-linear partial differential equation of the parabolic type, which describes the electric field $E$ as a function of time $t$ and distance $x$ from the electrode surface.

We will deal with the conditions right at the electrode surface independently, by appropriate boundary conditions.

\section{Static Solutions}

We will now solve equation 12 to get the electric field distribution $E$ under static, or equilibrium, conditions, that is, with the condition $\frac{\partial E}{\partial t}=0$. We will apply the solution to a half-cell where the electrode is in contact with an electrolyte containing its ions.

To do that, we will split the charge transfer reaction into two parts and treat the anodic and cathodic cases separately since they are essentially independently driven reactions. We will then get the resulting electric field $E$, representing the double-layer, by superimposing the anodic and cathodic field solutions $E_{a}$ and $E_{c}$.

In the anodic case, we will thus, in effect, be dealing with an electrode containing $\mathrm{Me}$-atoms interacting with an electrolyte void of electrode ions. 
In the cathodic case, we will, on the other hand, be effectively dealing with an electrolyte containing a concentration of a $a_{M e^{z+}} \mathrm{mol} / L$ electrode ions, interacting with an electrode now only a source of electrons.

We will utilize the electric field to calculate the electrode voltage $U$ as a function of electrode current and electrolyte activity relative to a reference half-cell. We will demonstrate that the result perfectly agrees with the Butler/Volmer and Nernst equations, which in turn strongly validates equation 12 and the associated electric field solutions.

We will also examine the resulting electric field $E$ as a function of electrode current.

\subsection{The Anodic Case}

The static solution to equation 12 is:

$$
E_{a}=-\frac{2 k T}{z e}\left(\frac{1}{x+x_{a}}\right)
$$

where $E_{a}$ is the electric field associated with the anodic case.

The $x_{a}$ parameter defines the electric field right at the electrode surface and is thus related to the electrode's ability to release ions into the electrolyte and, consequently, the electrode potential. It is also a measure of the thickness of the electric field distribution. The amplitude has fallen to one-half at that distance from the electrode surface.

Based on equation 8 the related electrode ion density distribution is given by $\rho_{a}$ as:

$$
\rho_{a}=\epsilon \frac{2 k T}{(z e)^{2}} \frac{1}{\left(x+x_{a}\right)^{2}}
$$

Figure 1 shows, as an example, the anodic electric field and the corresponding cation distribution for $x_{a}=10^{-6} \mathrm{~m}, z=2$ and $\epsilon=81 * \epsilon_{0}$.
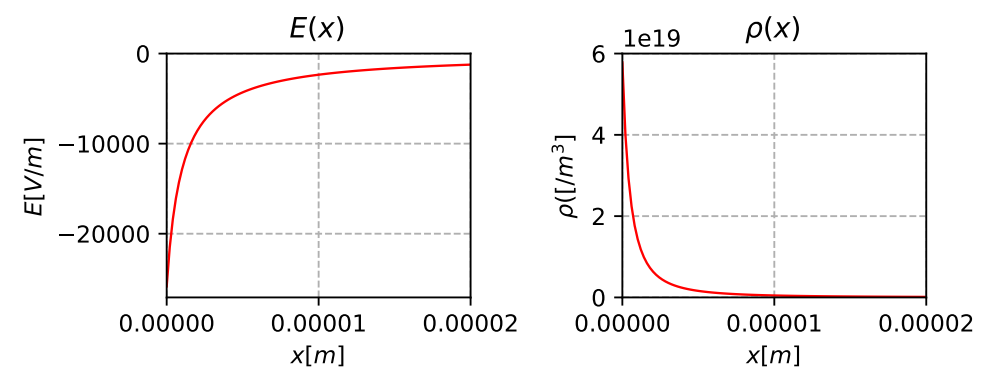

Figure 1: The anodic electric field and cation density, $\left(x_{a}=10^{-6} \mathrm{~m}\right)$.

We now need to define the proper boundary condition that relates the electric field at the electrode surface to the electrode current $I_{a}$. 
Typically, there is a linear relationship between electric field and current, both for electronic and electrolytic conductors, as illustrated by Ohm's Law. We will thus presume a simple linear relationship also in this case, as:

$$
I_{a}=- \text { const } \cdot E_{a}\left\lfloor_{x=0}\right.
$$

By inserting into equation 13 we obtain the following relation:

$$
I_{a}=\text { const } \cdot \frac{2 k T}{z e}\left(\frac{1}{x_{a}}\right)
$$

In order to be able to calculate the electrode voltage, we will need another half-cell as a reference electrode. Let us assume that it is identical to the half-cell we are studying, but with an electrolyte at a unit activity or $a_{r}=1$.

Similarly to equations 13,15 , and 16 , we have for the reference electrode:

$$
\begin{gathered}
E_{r a}=-\frac{2 k T}{z e}\left(\frac{1}{x+x_{r a}}\right) \\
I_{r a}=- \text { const } \cdot E_{r a}\lfloor x=0 \\
I_{r a}=\text { const } \cdot \frac{2 k T}{z e}\left(\frac{1}{x_{r a}}\right)
\end{gathered}
$$

We can now formulate the electrode voltage $U_{a}$ for our half-cell relative to the reference electrode, for the anodic case, as:

$$
U_{a}=-\frac{2 k T}{z e} \int_{\infty}^{0}\left(\frac{1}{x+x_{a}}-\frac{1}{x+x_{r a}}\right) d x
$$

which simplifies to:

$$
U_{a}=\frac{2 k T}{z e} \ln \left(\frac{x_{r a}}{x_{a}}\right)
$$

and:

$$
U_{a}=\frac{2 k T}{z e} \ln \left(\frac{I_{a}}{I_{r a}}\right)
$$

where $I_{r a}$ is the anodic current for the reference electrode.

Since we are assuming the reference electrode is in equilibrium condition, we have:

$$
I_{r a}=I_{r c}=i_{r}
$$

where $i_{r}$ is the exchange current for the reference electrode.

Thus:

$$
I_{a}=i_{r} e^{\frac{z e}{2 k T} U_{a}}
$$

which we recognice the anodic Tafel equation. 


\subsection{The Cathodic Case}

For the cathodic case, we will assume that the bulk of the electrolyte contains a concentration of $\rho_{c}$ electrode ions and that the electrode now is only a source of electrons. Since the electrode has virtually a limitless supply of free electrons available, we expect the concentration of ions right at the surface to be virtually zero. Thus the effective concentration will be $-\rho_{c}$ relative to the bulk of the electrolyte.

$$
\rho_{c}=-\epsilon \frac{2 k T}{(z e)^{2}} \frac{1}{\left(x+x_{c}\right)^{2}}
$$

With Avogadros Number $N_{A}$ we can convert $\rho_{c}$ to effective molarity, or activity $a_{M e^{z+}}$ :

$$
a_{M e^{z+}}=-\rho_{c} / N_{A}
$$

The boundary condition, in this case, will depend on both the ion activity $a_{M e^{z+}}$ and the electric field at the electrode surface $E_{c}$. We will again assume simple linear relationship for both, as:

$$
I_{c}=\text { const } \cdot a_{M e^{z+}} E_{c}\left\lfloor_{x=0}\right.
$$

At equilibrium, we also require:

$$
I_{a}=I_{c}=i_{o}
$$

where $i_{o}$ is the exchange current.

The cathodic electric field solution to equation 10 will have the opposite sign compared to the anodic case:

$$
E_{c}=\frac{2 k T}{z e}\left(\frac{1}{x+x_{c}}\right)
$$

Figure 2 shows, as an example the cathodic electric field and ion distribution for an electrolyte bulk molarity of $0.01 \mathrm{~mol} / \mathrm{L}$.
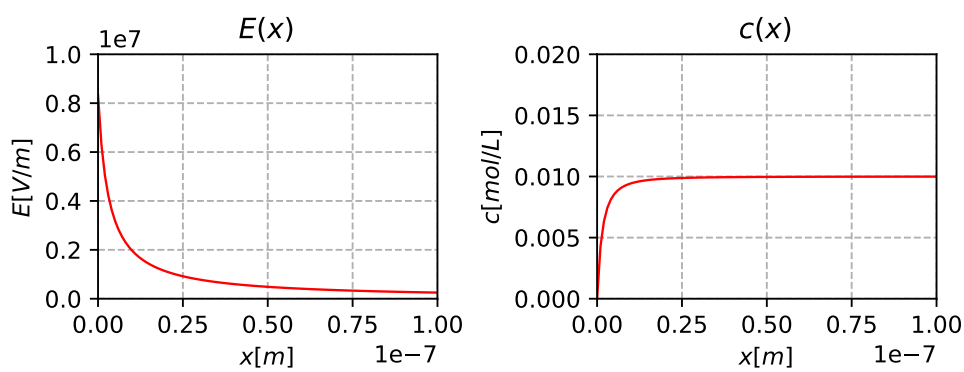

Figure 2: The cathodic electric field and ion concentration, (0.01mol in bulk). 
Equation 27 and 32 now yields:

$$
I_{c}=\text { const } \cdot a_{M e^{z+}} \frac{2 k T}{z e}\left(\frac{1}{x_{c}}\right)
$$

Similarly, for the reference electrode, we have:

$$
E_{r c}=\frac{2 k T}{z e}\left(\frac{1}{x+x_{r c}}\right)
$$

and:

$$
I_{r c}=\text { const } \cdot 1 \cdot \frac{2 k T}{z e}\left(\frac{1}{x_{r c}}\right)
$$

With the reference electrode at equilibrium, we have:

$$
I_{r c}=i_{r}
$$

Like before, for the anodic case, we can now formulate the electrode voltage for the cathodic case as:

$$
U_{c}=\int_{\infty}^{0} \frac{2 k T}{z e}\left(\frac{1}{x+x_{c}}-\frac{1}{x+x_{r c}}\right) d x
$$

which we can simplify to:

$$
U_{c}=\frac{2 k T}{z e} \ln \left(\frac{x_{c}}{x_{r c}}\right)
$$

Furthermore, related to the electrode current and the ion activity, as:

$$
U_{c}=\frac{2 k T}{z e} \ln \left(\frac{i_{r} a_{M e^{z+}}}{I_{c}}\right)
$$

Thus:

$$
I_{c}=i_{r} a_{M e^{z+}} e^{-\frac{z e}{2 k T} U_{c}}
$$

\subsection{Total Solution}

We will now combine the two solutions.

Since $U_{a}$ and $U_{c}$ refer to the same electrode voltage in the combined expression, we will replace both with $U$.

The total current $I$ is related to the electrode voltage, as follows:

$$
I=I_{a}-I_{c}=i_{r}\left(e^{\frac{z e e}{2 k T} U}-a_{M e^{z+}} e^{-\frac{z e}{2 k T} U}\right)
$$

By re-arranging the equation, we get:

$$
I=i_{r} \sqrt{a_{M e^{z+}}}\left(e^{\frac{z e}{2 k T}\left(U-\frac{k T}{z e} \ln \left(a_{M e^{z+}}\right)\right)}-e^{-\frac{z e}{2 k T}\left(U-\frac{k T}{z e} \ln \left(a_{M e^{z+}}\right)\right)}\right)
$$




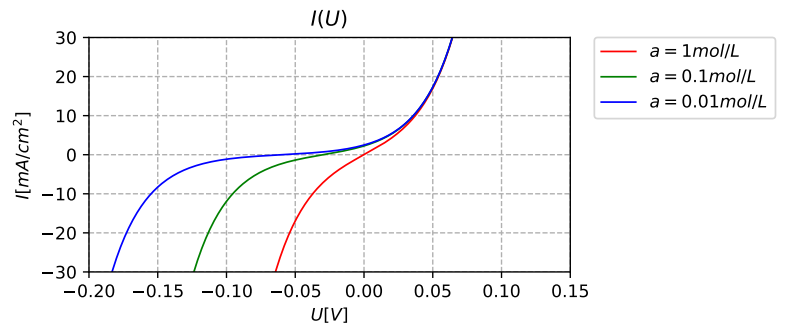

Figure 3: The half-cell DC current/voltage relationship $\left(i_{r}=2.5 \mathrm{~mA} / \mathrm{cm}^{2}, z=\right.$ 2).

By expressing the electrode voltage relative to the equilibrium voltage, which is traditionally called the overvoltage $(\eta)$, the expression takes this more familiar form:

$$
I=i_{0}\left(e^{\alpha \frac{z e}{k T} \eta}-e^{-(1-\alpha) \frac{z e}{k T} \eta}\right)
$$

which we recognize as the well-known Butler/Volmer equation with the transfer coefficient $\alpha=\frac{1}{2}$,

and where $i_{0}$ is the exchange current for the cell under study:

$$
i_{0}=i_{r} \sqrt{a_{M e^{z+}}}
$$

and where the overvoltage is:

$$
\eta=U-\frac{k T}{z e} \ln \left(a_{M e^{z+}}\right)
$$

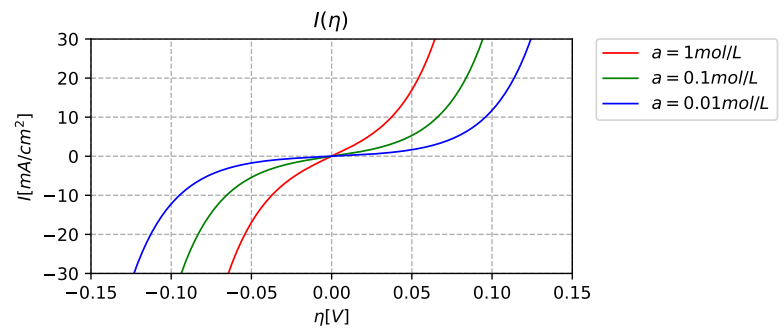

Figure 4: The Butler-Volmer relationship $\left(i_{r}=2.5 \mathrm{~mA} / \mathrm{cm}^{2}, z=2\right)$.

The theoretical basis for the introduction of the so-called transfer coefficient $\alpha$ by Butler and Volmer has been a debated issue, (Bauer, 1968). The traditional derivation of this equation predicts the transfer coefficient to be somewhere within a range between zero and one. Experimentally though, it is typically found to be close to 0.5 . With the present derivation, we note that simple linear boundary conditions at the electrode surface, predicts $\alpha=\frac{1}{2}$. 
For small deviations from equilibrium equation 40 simplifies to:

$$
I=i_{0} \frac{z e}{k T} \cdot \eta
$$

or:

$$
R_{0}=\frac{k T}{z e} \cdot \frac{1}{i_{0}}
$$

where $R_{0}$ is the DC resistance of the cell or the transfer reaction resistance.

At equilibrium, with no external electrode current we have:

$$
U=\frac{k T}{z e} \ln \left(a_{M e^{z+}}\right)
$$

Let us now use a different electrode, characterized by $x_{r b}$, in the reference half-cell in contact with an electrolyte containing its ions at unit activity.

We have then:

$$
E_{b}=-\frac{2 k T}{z e}\left(\frac{1}{x+x_{r b}}\right)
$$

where $E_{b}$ is the anodic electric field for the reference half-cell.

Similar to equation 21 we now have the electrode potential for our half-cell with respect to the new reference half-cell (we assume here the valence for the two electrode reactions are the same):

$$
E^{0}=\frac{2 k T}{z e} \ln \left(\frac{x_{r b}}{x_{a}}\right)
$$

If we regard our reference half-cell as our standard reference electrode, this equation modifies to the predictions of Nernst's equation:

$$
U=E^{0}+\frac{k T}{z e} \ln \left(a_{M e^{z+}}\right)
$$

where $E^{0}$ is the standard electrode potential for the electrode under study when immersed in an electrolyte containing its ions at unit activity.

We need here to point out that equation 47 is valid only if there is no saturation of electrode ions at the electrode surface. This will be discussed further in Section 4.

\subsubsection{The Electric Field at the Electrode/Electrolyte Interface}

We will now examine the electric field in the electrolyte associated with the charge transfer reaction.

At equilibrium, by definition, the anodic and cathodic exchange currents are equal; thus, $I_{a}=I_{c}$. 
The corresponding anodic and cathodic electric field distributions are then also equal in amplitude but with opposite signs. The resultant field thus cancels out.

Let us introduce the parameter $x_{0}$ to designate the anodic and cathodic electric field thicknesses at equilibrium.

With equations 16 and 30 thus follows:

$$
x_{0}=x_{a}=x_{c}
$$

We will now supply an external current $I$ to the electrode and derive the resulting electric field distribution. We will treat the anodic and cathodic current components separately and then add the corresponding electric field components together to get the total electric field $E(x)$,

Separating equation 40 into its anodic and cathodic current components we have:

$$
\eta=\frac{2 k T}{z e} \ln \left(\frac{I_{a}}{i_{0}}\right)=-\frac{2 k T}{z e} \ln \left(\frac{I_{c}}{i_{0}}\right)
$$

and thus:

$$
I_{a} I_{c}=i_{0}^{2}
$$

Let $I_{a}=I+i_{0}$, then we have $I_{c}=\frac{i_{0}^{2}}{i_{0}+I}$ for $I>=0$

We can now formulate the electrode current's influence on the anodic and cathodic electric field thicknesses as:

$$
\begin{aligned}
& x_{a}=x_{0} \frac{i_{0}}{i_{0}+I} \\
& x_{c}=x_{0} \frac{i_{0}+I}{i_{0}}
\end{aligned}
$$

Finally, we have the total electric field $E(x)$ for $I>=0$ :

$$
E(x)=E_{a}+E_{c}=-\frac{2 k T}{z e}\left[\frac{1}{x+x_{a}}-\frac{1}{x+x_{c}}\right]
$$

With $I<0$ we have $I_{c}=-I+i_{0}$ and $I_{a}=\frac{i_{0}^{2}}{i_{0}-I}$ and also:

$$
\begin{aligned}
& x_{c}=x_{0} \frac{i_{0}}{i_{0}-I} \\
& x_{a}=x_{0} \frac{i_{0}-I}{i_{0}}
\end{aligned}
$$

Thus, the total electric field $E(x)$ for $I<0$ :

$$
E(x)=\frac{2 k T}{z e}\left[\frac{1}{x+x_{a}}-\frac{1}{x+x_{c}}\right]
$$

Figure 5 shows the calculated electric field for a range of electrode currents 


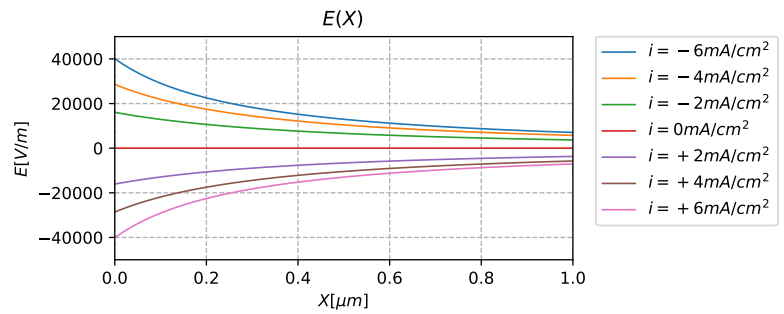

Figure 5: The Electric Field, E(x) $\left(x_{0}=1 e-6, i_{0}=2.5 \mathrm{~mA} / \mathrm{cm}^{2}, z=2, a_{M e^{z+}}=\right.$ $1 \mathrm{~mol} / L)$.

\section{Dynamic Solutions}

The theoretical model described by equation 12 allows us also to evaluate the electrode voltage in non- equilibrium situations - thus when $\frac{\partial E}{\partial t} \neq 0$.

As a first example, we will calculate the electrode voltage as a function of time from the moment an electrode makes contact with an electrolyte void of electrode ions.

In the second example, we will calculate the electrode voltage as a function of time from when an electrode contacts an electrolyte containing electrode ions.

In the third example of dynamic solutions, we will calculate the half cell's dynamic, or complex, impedance.

\subsection{Electrode in Inert Electrolyte}

We will assume that the electric field at the electrode surface will be established and remain constant from when we dip the electrode into the electrolyte. Since equation 15 states this assumption, we will use it as both initial and boundary conditions.

We are now ready to state the problem as follows:

$$
\begin{cases}\frac{\partial E}{\partial t}=D\left(\frac{\partial^{2} E}{\partial x^{2}}-\frac{z e}{k T} E \frac{\partial E}{\partial x}\right) \\ E(x, 0)=0, & (0<x) \\ E(0, t)=-\frac{2 k T}{z e x_{a}}, & (0<t) \\ U(t)=\int_{0}^{t} E d x & \end{cases}
$$

Due to the non-linear nature of equation 12 it is not possible to solve for the electrode potential analytically. However, we can split the problem into two parts. The initial part of the electrode voltage response can be solved numerically. The latter part we will solve via an analytical approach, which will extend the initial response to late time. 


\subsubsection{Numerical Solution to the Initial Response}

In order to make the numerical process efficient, we will make the following substitution:

$$
x+x_{0}=x_{0} e^{y}
$$

This substitution will compress the spatial range, allowing us to speed up the numerical process significantly.

The statement of the problem will now be as follows:

$$
\left\{\begin{array}{l}
\frac{\partial E}{\partial t}=\frac{D}{\left(x_{0} e^{y}\right)^{2}}\left(\frac{\partial^{2} E}{\partial y^{2}}-\left(1+\frac{z e}{k T} x_{0} e^{y} E\right) \frac{\partial E}{\partial y}\right) \\
E(y, 0)=0, \quad(0<y) \\
E(0, t)=-\frac{2 k T}{z e x_{0}}, \quad(0<t) \\
U(t)=\int_{0}^{t} E x_{0} e^{y} d y
\end{array}\right.
$$

We will approximate the continuous function $E(y, t)$ with a function that is defined only at the grid points $t=n \Delta t$ and $y=n \Delta y$, and designate it as $E_{i, n}$.

We will utilize the Crank-Nicolson scheme to achieve a correct second-order approximation for the spatial derivatives at grid points $\left(i, n+\frac{1}{2}\right)$, as shown below:

$$
\begin{aligned}
\frac{E_{i, n+1}-E_{i, n}}{\Delta t} & =\frac{D}{\left(x_{0} e^{i \Delta y}\right)^{2}}\left[\frac{E_{i+1, n}-2 E_{i, n}+E_{i-1, n}+E_{i+1, n+1}-2 E_{i, n+1}+E_{i-1, n+1}}{2(\Delta y)^{2}},\right. \\
& \left.-\left(1+\frac{z e}{k T} x_{0} e^{i \Delta y} E_{i, n+1}\right) \frac{E_{i+1, n}-E_{i-1, n}+E_{i+1, n+1}-E_{i-1, n+1}}{4 \Delta y}\right]
\end{aligned}
$$

In order to make this a linear system of equations, we will express the field $E_{i, n+\frac{1}{2}}$ with the function values at the known instances of time $n \Delta t$.

Therefore, we will carry out a Taylor expansion centered on grid point $(i, n)$. This results in:

$$
E_{i, n+\frac{1}{2}} \approx E_{i, n}+\left(\frac{\partial E}{\partial t}\right)_{i, n} \cdot\left(\frac{\Delta t}{2}\right)+\left(\frac{\partial^{2} E}{\partial x^{2}}\right)_{i, n} \cdot \frac{1}{2 !}\left(\frac{\Delta t}{2}\right)^{2}+\cdots
$$

Thus, with equation 61 we get:

$$
\begin{aligned}
E_{i, n+\frac{1}{2}}= & E_{i, n}+\frac{D \Delta t}{2\left(x_{0} e^{i \Delta y}\right)^{2}}\left[\frac{E_{i+1, n}-2 E_{i, n}+E_{i-1, n}}{(\Delta y)^{2}},\right. \\
& \left.-\left(1+\frac{z e}{k T} x_{0} e^{i \Delta y} E_{i, n}\right) \frac{E_{i+1, n}-E_{i-1, n}}{2 \Delta y}\right]
\end{aligned}
$$

Equation 61 can now be formulated as a linear system of equations as shown below: 


$$
\begin{array}{r}
(1+H) E_{i-1, n+1}+(-2-I) E_{i, n+1}+(1-H) E_{i+1, n+1}, \\
\left.\quad=(-1-H) E_{i-1, n}+(2-I) E_{i, n}+(-1+H) E_{i+1, n}\right)
\end{array}
$$

where:

$$
H=\frac{\Delta y}{2}\left(1+\frac{z e}{k T} x_{0} e^{i \Delta y} E_{i, n+\frac{1}{2}}\right)
$$

and:

$$
\left.I=\frac{2\left(\Delta y x_{0} e^{i \Delta y}\right)^{2}}{D \Delta t}\right)
$$

For $i=0$, that is, at the electrode surface:

$$
E(0, n)=\frac{2 k T}{z e} \frac{1}{x_{0}}
$$

Before we can calculate $E_{i, n+1}$, we need to determine the step sizes $\Delta t$ and $\Delta y$, the number of time-steps $N t$ and spatial steps $N y$.

For each new $n$ we will have a system of equations of the following type:

$$
a_{i} E_{i-1}+b_{i} E_{i}+c_{i} E_{i+1}=d_{i}
$$

where $(1<=i<=N y)$ and $a_{1}=c_{N y}=0$

The coefficients form a tri-diagonal matrix, which allows us to use Thomas efficient algorithm to solve the system of equations, as shown below:

First, we calculate:

$$
\beta_{i}=b_{i}-\frac{a_{i} c_{i-1}}{\beta_{i-1}}, \text { with } \beta_{1}=b_{1}
$$

and then:

$$
\gamma_{i}=\frac{d_{i}-a_{i} \gamma_{i-1}}{\beta_{i}}, \text { with } \gamma_{1}=\frac{d_{1}}{b_{1}}
$$

The requested function $E_{i, n}$ is now obtained as:

$$
E_{i, n}=\gamma_{i}-\frac{c_{i} E_{i+1}}{\beta_{i}} \text { with } E_{N y}=\gamma_{N y}
$$

The initial portion of electrode voltage response is obtained numerically as:

$$
U(t)=\frac{1}{2} E_{0, n} \Delta y x_{0}+\sum_{i=1}^{N y} E_{i, n} x_{0} e^{y} \Delta y
$$

(With electrode voltage, in this case, we mean the voltage relative to the electrolyte - which we cannot measure directly. In practice, we would use a suitable reference electrode.) 


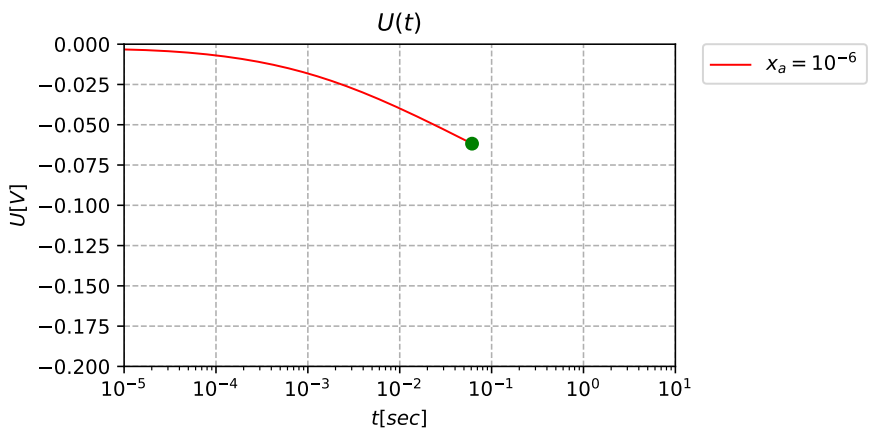

Figure 6: The initial response $\left(x_{a}=10^{-6} \mathrm{~m}, z=2, D=6 \cdot 10^{-10}\right)$

\subsubsection{Analytical Extension to the Initial Response}

It is possible to find an asymptotically converging function $\varphi(t)$ to the electrode voltage $U(t)$ that will allow us to extend the result of the numerical solution to late times.

As a starting point, we will use the differential equation 60 :

$$
\frac{\partial E}{\partial t}=\frac{D}{\left(x_{a} e^{y}\right)^{2}}\left(\frac{\partial^{2} E}{\partial y^{2}}-\left(1+\frac{z e}{k T} x_{a} e^{y} E\right) \frac{\partial E}{\partial y}\right)
$$

By making the substitution $S=\frac{t D}{x_{a}^{2}}$ we get:

$$
\frac{\partial E}{\partial S}=e^{-2 y}\left(\frac{\partial^{2} E}{\partial y^{2}}-\left(1-2 \frac{E}{E_{\infty}}\right) \frac{\partial E}{\partial y}\right)
$$

where $E_{\infty}$ is the stationary field solution according to equation 15 .

By making the substitution $V=\frac{E}{E_{\infty}}$ we get:

$$
\frac{\partial V}{\partial S}=e^{-2 y}\left(\frac{\partial^{2} V}{\partial y^{2}}-2 \frac{\partial V}{\partial y}+V-(1-2 V)\left(\frac{\partial V}{\partial y}-V\right)\right)
$$

With equation 13 and the substitution in equation 59 we have:

$$
E_{\infty}=-\frac{2 k T}{z e x_{a}} e^{-y}
$$

We can now formulate the electrode potential as:

$$
\begin{aligned}
U(t) & =-\frac{k T}{z e} \int_{0}^{\infty} \frac{2 e^{-y}}{x_{a}} V(y, S) d x \\
& =-\frac{k T}{z e} \int_{0}^{\infty} 2 V(y, S) d y \\
& =-\frac{k T}{z e} f\left(\frac{D t}{x_{a}^{2}}\right)
\end{aligned}
$$


Where the function $f$ is the solution to the integral.

Using equation 15 we can now solve for the function $f$ as follows:

$$
\left\{\lim _{t \rightarrow \infty} \frac{\partial\left(-\frac{k T}{z e} f\left(\frac{D t}{x_{a}^{2}}\right)\right)}{\partial x_{a}}\right\} \equiv\left\{\lim _{x_{1} \rightarrow \infty} \frac{\partial\left(-\frac{2 k T}{z e} \int_{0}^{x_{1}} \frac{d x}{x+x_{a}}\right)}{\partial x_{a}}\right\}
$$

which results in:

$$
f^{\prime}\left(\frac{D t}{x_{a}^{2}}\right)=\frac{x_{a}^{2}}{D t}
$$

and thus:

$$
f\left(\frac{D t}{x_{a}^{2}}\right)=\ln \left(\frac{D t}{x_{a}^{2}}\right)+C_{1}
$$

Finally we have an expression for the function $\varphi(t)$ as follows:

$$
\varphi(t)=-\frac{k T}{z e} \ln \left(\frac{D t}{x_{a}^{2}}\right)+C_{1}
$$

which asymptotically converges towards the late-time electrode potential response $U(t)$.

Since $t$ is the only variable, the slope of $\varphi(t)$ is $29.7 \mathrm{mV}$ per decade for $z=2$, as an example.

\subsubsection{Total Solution}

We can now provide a complete solution to the electrode potential response from the moment we dip the electrode into the electrolyte - to late times. We need to extend the numerical solution sufficiently far in time so that $\varphi(t)$ can take over. We can determine the constant $C_{1}$ at the attachment points of the two partial solutions.

Figure 7 shows the combined solution, where the green dot shows the transition point from the numerical to the analytical solution.

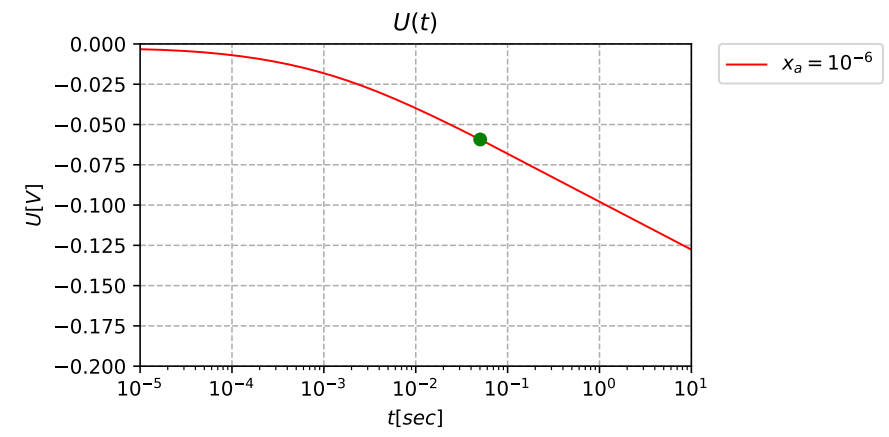

Figure 7: The combined numerical and analytical solution 
Figure 8 shows the combined solution for a range of $x_{a}$ parameters

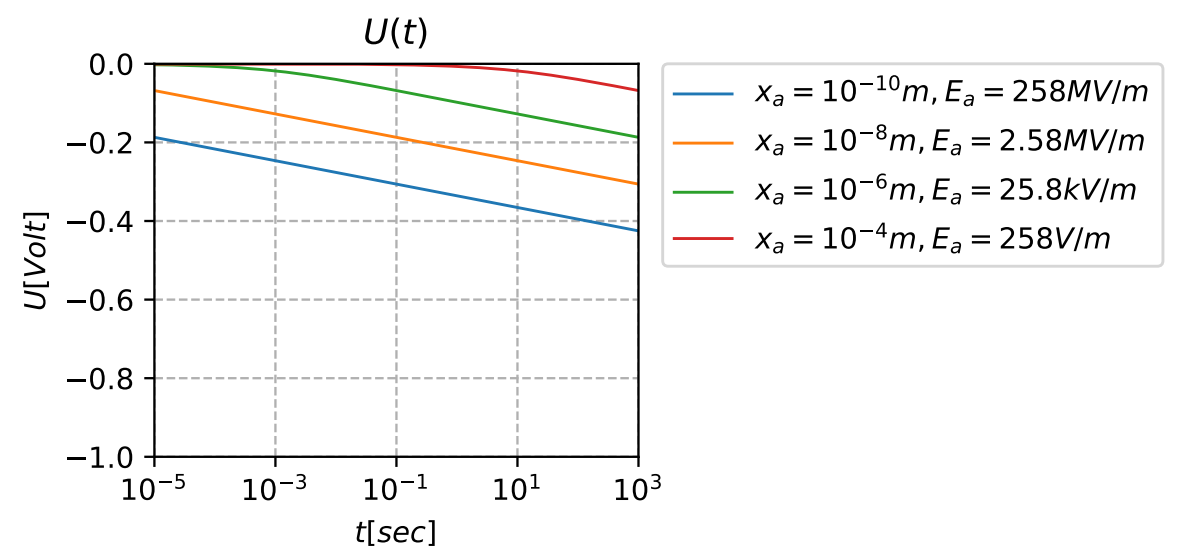

Figure 8: The total response for different $x_{a}$ parameters

\subsubsection{Saturation}

It is essential to realize that when the size of the $x_{a}$ parameter gets smaller, the electrode ion concentration at the surface increases. At some point, the concentration gets saturated because of a lack of room.

According to equation 14 we have following relationship at the electrode surface:

$$
x_{a}=\sqrt{\frac{2 \epsilon k T}{\rho(z e)^{2}}}
$$

From equation 82 we also note that $x_{a}$ gets smaller when $\rho$ increases to the point that when, as an example, $\rho=3 \cdot 10^{28}$ then $x_{a}=0.044 n m$, which gets to be smaller than the size of the electrode ions.

We will here make the simple assumption that the saturation level, $\rho_{\text {sat }}$, is $5 \cdot 10^{27} / \mathrm{m}^{3}$, which corresponds to $x_{a}=10^{-10} \mathrm{~m}$.

Figure 10 shows the electrode potential response for a range of thicknesses of the saturated layer.

\subsubsection{Experimental Evaluation}

We will here compare the calculated electrode voltage response with actual measurements, where a zinc electrode (Zinc rod $99.998 \%, 7 \mathrm{~mm}$ diam.),is dipped into an $0.1 \mathrm{M} \mathrm{Na}_{2} \mathrm{SO}_{4}$ electrolyte.

We measured the electrode potential relative to a Calomel reference electrode using a high-impedance digital voltmeter, over a range of times from 5 to 640 sec after the electrode made the initial contact with the electrolyte. 

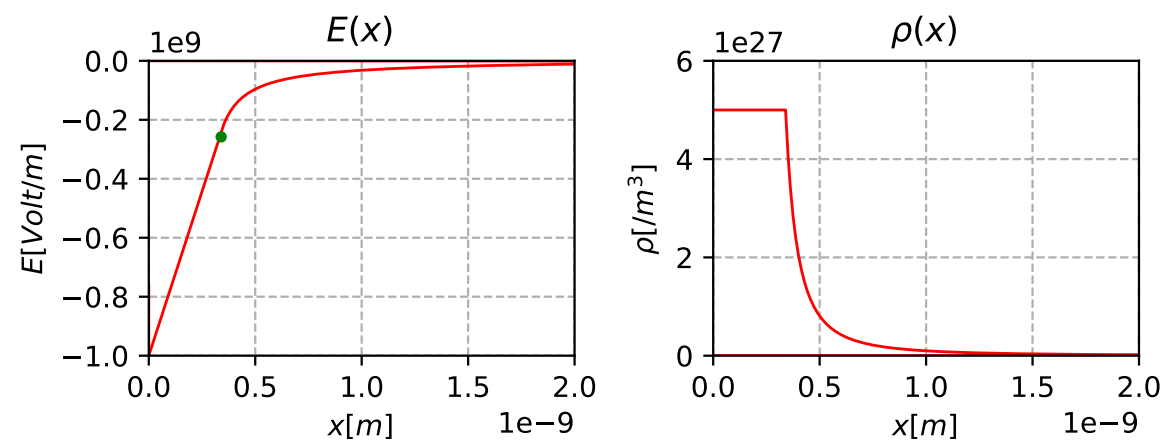

Figure 9: Electric field and Ion density. Saturation.

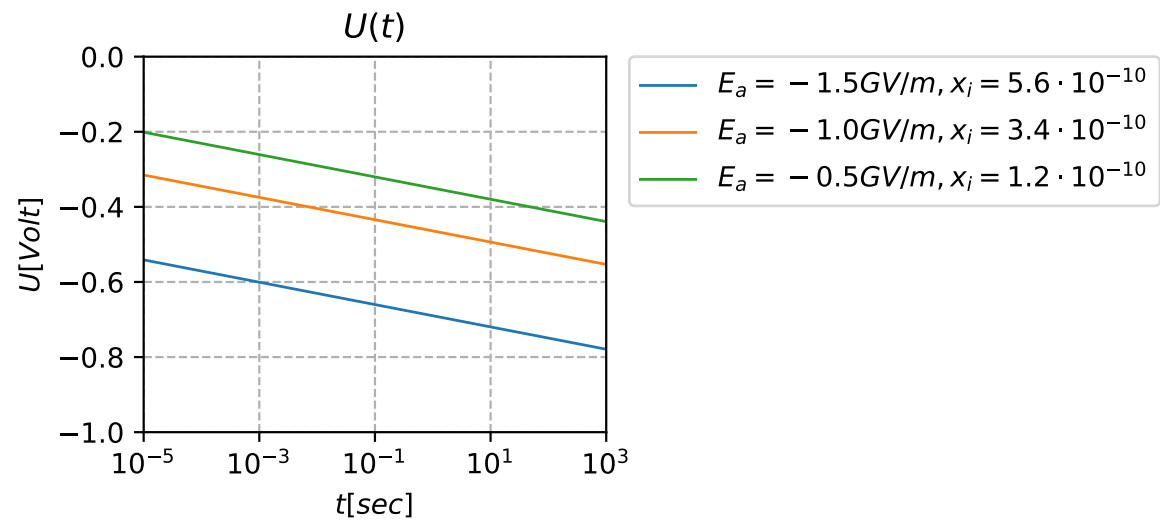

Figure 10: Saturated Potential Decays.

Since we deal with a surface phenomenon, we need to ensure that the electrode surface is clean. The following procedure resulted in repeatable measurements: etching of the electrode in diluted $H C L$ followed by thorough cleaning in distilled water

First of all, we notice in figure 11 that, in general, the measured electrode voltages are well below even those corresponding to $x_{a}=10^{-10} \mathrm{~m}$, as shown in figure 8 , which points to a saturated layer of ions next to the electrode surface, or a thin surface layer of the electrode void of valence electrons - thus positively charged.

Based on the assumed saturation level of $\rho_{\text {sat }}=5 \cdot 10^{27} / \mathrm{m}^{3}$, the best fit is obtained at an electrode surface field strength of $1.56 \mathrm{GV} / \mathrm{m}$. The corresponding thickness, $x_{i}$, of the saturated layer is then $x_{i}=5.9 \cdot 10^{-10} \mathrm{~m}$. The saturated layer establishes itself very quickly since it is very close to the electrode - or even part of it. 


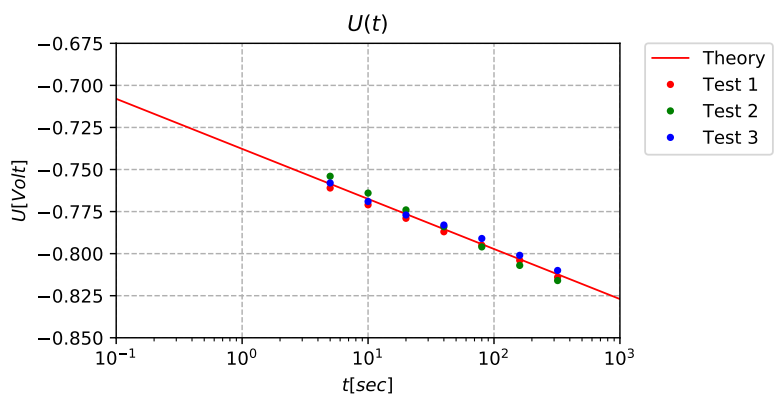

Figure 11: Test results

The diffuse portion, on the other hand, will gradually evolve with time.

As is evident from the graph, the experimental results confirm the theory's prediction of the diffuse layer's gradual formation with a high degree of certainty. We are well beyond the initial response; thus, the analytical extension provides the best fit.

We also notice that the initially assumed boundary condition related to the electric field at the electrode surface is constant appears valid and that the anodic exchange current is indeed time-independent.

\subsection{Electrode in Electrolyte containing its Ions}

When an electrode contacts an electrolyte containing a certain concentration of electrode ions, it will take some time for the electrode potential to stabilize and establish equilibrium with the electrolyte. We will here calculate the electrode voltage response from contact until equilibrium. We will carry out this calculation for a range of ion densities.

First, we will calculate the cathodic voltage response caused by ions in the electrolyte bulk recombining with electrode electrons.

As a next step, we will combine this cathodic reaction with the anodic, which we already calculated in the previous section.

We expect that, after an initial transient, the electrode voltage will eventually stabilize - and end up at the equilibrium voltage predicted by Nernst's Equation.

The problem statement will be similar to the statements in equation 60 , except for the boundary condition. Like what was done in equation 25 , as a boundary condition, we will require the bulk ion density $\rho$ to become zero at the electrode surface. We will, though, use here the equivalent condition of a density of $-\rho$ at the electrode surface and zero well away. By using Gauss Law, as in equation 8 , we can express this condition in terms of the electric field. However, in order to provide a second-order correct electric field derivative at the electrode surface, as a consequence of the specified boundary condition, we will move the location of the electrode surface to $i=\frac{1}{2}$. 
The statement of the problem is thus as follows:

$$
\left\{\begin{array}{l}
x_{c}=\sqrt{\frac{2 \epsilon k T}{\rho(z e)^{2}}} \\
\frac{\partial E}{\partial t}=\frac{D}{\left(x_{c} e^{y}\right)^{2}}\left(\frac{\partial^{2} E}{\partial y^{2}}-\left(1+\frac{z e}{k T} x_{c} e^{y} E\right) \frac{\partial E}{\partial y}\right) \\
E(y, 0) \equiv 0, \quad(0<y) \\
\frac{\partial E(0, t)}{\partial y}=\rho \frac{z e x_{c}}{\epsilon}, \quad(0<t) \\
U(t)=\int_{0}^{t} E x_{c} e^{y} d y
\end{array}\right.
$$

\subsubsection{Numerical Solution}

The numerical solution to the problem follows the procedures in Section 4.1.1 except as shown below:

$$
H=\frac{\Delta y}{2}\left(1+\frac{z e}{k T} x_{c} e^{\left(i-\frac{1}{2}\right) \Delta y} E_{i, n+\frac{1}{2}}\right)
$$

and:

$$
\left.I=\frac{2\left(\Delta y x_{c} e^{\left(i-\frac{1}{2}\right) \Delta y}\right)^{2}}{D \Delta t}\right)
$$

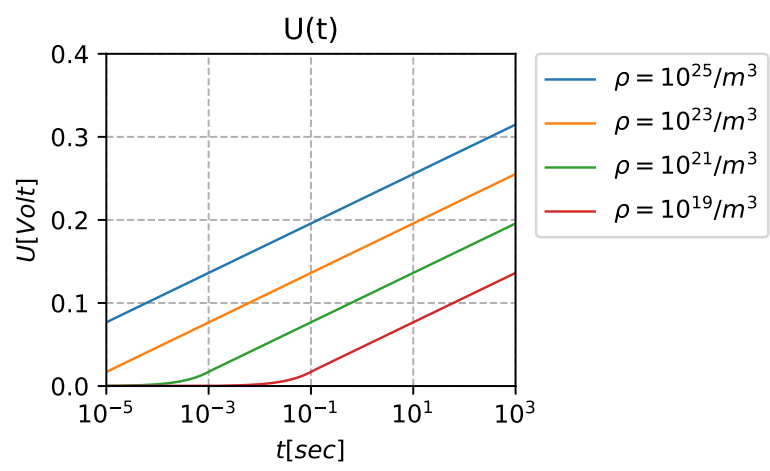

Figure 12: Cathodic Electrode Voltage $(\mathrm{z}=2)$

$$
\begin{gathered}
E_{i, n+\frac{1}{2}}=E_{i, n}+\frac{D \Delta t}{2\left(x_{c} e^{\left(i-\frac{1}{2}\right) \Delta y}\right)^{2}}\left[\frac{E_{i+1, n}-2 E_{i, n}+E_{i-1, n}}{(\Delta y)^{2}},\right. \\
\left.-\left(1+\frac{z e}{k T} x_{c} e^{\left(i-\frac{1}{2}\right) \Delta y} E_{i, n}\right) \frac{E_{i+1, n}-E_{i-1, n}}{2 \Delta y}\right] \\
\frac{E_{1, n}-E_{0, n}}{\delta y}=a \frac{z e x_{c}}{\epsilon}
\end{gathered}
$$




$$
\begin{array}{r}
{[-1+H-I] E_{1, n+1}+[1-H] E_{2, n+1}=[1-H-I] E_{1, n},} \\
+[-1+H] E_{2, n}+2 \Delta y \frac{z e x_{c} a}{\epsilon}[1+H]
\end{array}
$$

Figure 12 shows the resulting solution to the cathodic process for a range of concentrations.

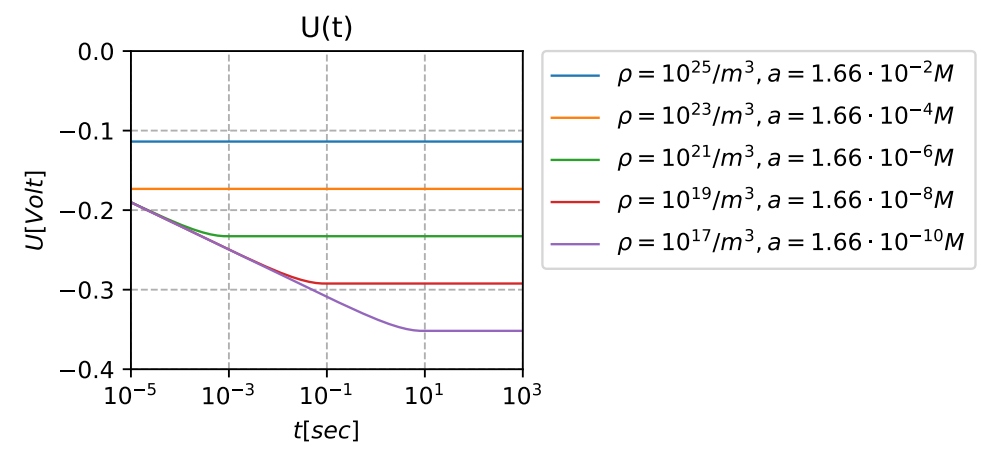

Figure 13: Total Electrode Voltage $\left(z=2, x_{a}=10^{-10}\right)$

Figure 13 shows the electrode voltage for the combined anodic and cathodic processes.

We observe that reaching equilibrium will take some time; the lower the concentration, the longer it will take.

The previous section shows that equilibrium will never occur if the concentration is zero.

With electrode voltage, in this case, we mean the voltage relative to the electrolyte - which we cannot measure directly. In practice, we would use a suitable reference electrode.

Let us assume we use as a reference electrode, a half-cell with an electrode with $x_{a}=10^{-10}$, and with an electrolyte activity of $1.66 \cdot 10^{-2}$. It would thus be at the same potential as the blue plot in Figure 13.

We observe that the voltage difference between pairs of plots at late times is $59 \mathrm{mV}$, which we expect for concentration ratios of two orders of magnitude.

The orange plot is thus at $-59 \mathrm{mV}$. The green plot is twice that, and so on.

The fully established equilibrium voltage predictions at late times thus agree with Nernst's equation.

\subsection{Complex Electrode Impedance}

The task here is to derive the complex impedance of the electrode/electrolyte interface reaction based on equation 12 .

Since the interface reaction, and thus the equation is non-linear, the transfer impedance will be linear only for amplitude within, let say $\pm 0.01 \mathrm{~V}$ from the equilibrium potential. We will therefore restrict the analysis to that region. 
Since the half-cell is at equilibrium, we only need to deal with either the anodic or cathodic part of the reaction. The contribution to the impedance from the other part of the reaction will be the same.

A linear solution must be of the form:

$$
E(x, t)=E_{1}(x)+E_{2}(x) e^{j \omega t}
$$

Inserting this into equation 12 yields:

$$
\frac{j \omega}{D} E_{2} e^{j \omega t}=\frac{\partial^{2} E_{1}}{\partial x^{2}}+\frac{\partial^{2} E_{2}}{\partial x^{2}} e^{j \omega t}-\frac{z e}{k T}\left(\left(E_{1}+E_{2} e^{j \omega t}\right)\left(\frac{\partial E_{1}}{\partial x}+\frac{\partial E_{2}}{\partial x} e^{j \omega t}\right)\right)
$$

which can be separated into a static equation for $E_{1}$ :

$$
\frac{\partial^{2} E_{1}}{\partial x^{2}}-\frac{z e}{k T} E_{1} \frac{\partial E_{1}}{\partial x}=0
$$

and a dynamic equation for $E_{2}$ :

$$
\left.\frac{j \omega}{D} E_{2}=\frac{\partial^{2} E_{2}}{\partial x^{2}}-\frac{z e}{k T}\left(\left(E_{1} \frac{\partial E_{2}}{\partial x}\right)+E_{2} \frac{\partial E_{1}}{\partial x}\right)\right)
$$

By inserting the solution to the equation 91 for $E_{1}$ and by eliminating the second harmonic, since we are restricting the analysis to the linear case, we finally obtain the following equation, which will allow us to solve for the complex impedance.

$$
\frac{\partial^{2} E_{2}}{\partial x^{2}}+\frac{2}{x+x_{0}} \frac{\partial E_{2}}{\partial x}-\left(\frac{2}{\left(x+x_{0}\right)^{2}}+j \frac{\omega}{D}\right) E_{2}=0
$$

Let us now make the following substitution:

$$
-j \frac{\omega}{D}\left(x+x_{0}\right)^{2}=y^{2}
$$

This results in:

$$
y^{2} \frac{\partial^{2} E_{2}}{\partial y^{2}}+2 y \frac{\partial E_{2}}{\partial y}+\left(y^{2}-2\right) E_{2}=0
$$

Another useful substitution:

$$
E_{2}=\frac{e_{2}}{\sqrt{y}}
$$

results in:

$$
y^{2} \frac{\partial^{2} e_{2}}{\partial y^{2}}+y \frac{\partial e_{2}}{\partial y}+\left(y^{2}-2 \frac{1}{4}\right) e_{2}=0
$$

which is a Bessel Equation with the solution:

$$
e_{2}=A \cdot J_{1.5}(y)+B \cdot J_{-1.5}(y)
$$


We require $E(y) \rightarrow 0$ when $y \rightarrow \infty$, thus follows:

$$
E_{2}=\frac{A}{\sqrt{y}} \cdot\left(J_{1.5}(y)-j J_{-1.5}(y)\right)
$$

We can express this equation in elementary function as:

$$
\begin{aligned}
& \operatorname{Re}\left(E_{2}\right)=A \frac{e^{-\gamma z}}{z}\left(\frac{\cos (\gamma z)}{z}+\sqrt{2} \cdot \gamma \cdot \sin \left(\gamma z+\frac{\pi}{4}\right)\right) \\
& \operatorname{Im}\left(E_{2}\right)=A \frac{e^{-\gamma z}}{z}\left(\frac{\sin (\gamma z)}{z}-\sqrt{2} \cdot \gamma \cdot \cos \left(\gamma z+\frac{\pi}{4}\right)\right)
\end{aligned}
$$

where $\gamma=\sqrt{\frac{\omega}{2 D}}$ and $z=x+x_{0}$.

We now have:

$$
\begin{aligned}
\operatorname{Re}\left(E_{2}\right)= & A \cdot e^{-\sqrt{\frac{\omega}{2 D}}\left(x+x_{0}\right)}\left[\frac{\cos \left(\sqrt{\frac{\omega}{2 D}}\left(x+x_{0}\right)\right)}{\left(x+x_{0}\right)^{2}},\right. \\
& \left.+\frac{\sqrt{2} \cdot \sqrt{\frac{\omega}{2 D}}}{x+x_{0}} \sin \left(\sqrt{\frac{\omega}{2 D}}\left(x+x_{0}\right)+\frac{\pi}{4}\right)\right] \\
\operatorname{Im}\left(E_{2}\right)= & A \cdot e^{-\sqrt{\frac{\omega}{2 D}}\left(x+x_{0}\right)}\left[\frac{\sin \left(\sqrt{\frac{\omega}{2 D}}\left(x+x_{0}\right)\right)}{\left(x+x_{0}\right)^{2}},\right. \\
& \left.-\frac{\sqrt{2} \cdot \sqrt{\frac{\omega}{2 D}}}{x+x_{0}} \cos \left(\sqrt{\frac{\omega}{2 D}}\left(x+x_{0}\right)+\frac{\pi}{4}\right)\right]
\end{aligned}
$$

Let us now define the amplitude of $E_{2}$ at the electrode surface to be $E_{20}$. Also, as stated before, we assume that this field is proportional to the current at the interface. Thus:

$$
E_{20}(j \omega t)=E_{2}(j \omega t)\left\lfloor_{x=0}=\text { const } \cdot i(j \omega t)\right.
$$

At the boundary, the electric field has to be real. The coordinate system will therefore have to be rotated by the amount $\varphi$ so that the imaginary component goes to zero. We therefore have:

$$
\varphi=-\operatorname{arctg}\left(\frac{\operatorname{Im}\left(E_{2}\right)}{\operatorname{Re}\left(E_{2}\right)}\left\lfloor_{x=0}\right)\right.
$$

and:

$$
\varphi=\operatorname{arctg}\left(\frac{\sqrt{2} \cdot \alpha \cdot \cos \left(\alpha+\frac{\pi}{4}\right)-\sin (\alpha)}{\sqrt{2} \cdot \alpha \cdot \sin \left(\alpha+\frac{\pi}{4}\right)+\cos (\alpha)}\right)
$$

Let's now make following substitutions $\frac{x}{x_{0}}=y$, and:

$$
\sqrt{\frac{\omega}{2 D}} \cdot x_{0}=\alpha
$$


We will then obtain:

$$
\begin{aligned}
\operatorname{Re}\left(E_{2}\right)= & A \cdot e^{-\alpha(1+y)}\left[\frac{\cos (\alpha(1+y)+\varphi)}{(1+y)^{2}},\right. \\
& \left.+\frac{\sqrt{2} \cdot \alpha}{1+y} \sin \left(\alpha(1+y)+\varphi+\frac{\pi}{4}\right)\right] \\
\operatorname{Im}\left(E_{2}\right)= & A \cdot e^{-\alpha(1+y)}\left[\frac{\sin (\alpha(1+y)+\varphi)}{(1+y)^{2}},\right. \\
& \left.-\frac{\sqrt{2} \cdot \alpha}{1+y} \cos \left(\alpha(1+y)+\varphi+\frac{\pi}{4}\right)\right]
\end{aligned}
$$

At the boundary we now have:

$$
E_{20}=A \cdot e^{-\alpha}\left[\cos (\alpha+\varphi)+\sqrt{2} \alpha \cdot \sin \left(\alpha+\varphi+\frac{\pi}{4}\right)\right]
$$

We are now able to calculate the electrode voltage as:

$$
\begin{aligned}
\operatorname{Re}(u)= & \frac{E_{20}}{\cos (\alpha+\varphi)+\sqrt{2} \alpha \cdot \sin \left(\alpha+\varphi+\frac{\pi}{4}\right)} \int_{0}^{\infty} e^{-\alpha y\left[\frac{\cos (\alpha(1+y)+\varphi)}{(1+y)^{2}}\right.}, \\
& \left.+\frac{\sqrt{2} \cdot \alpha}{1+y} \sin \left(\alpha(1+y)+\varphi+\frac{\pi}{4}\right)\right] d y
\end{aligned}
$$

$$
\begin{aligned}
\operatorname{Im}(u)= & \frac{E_{20}}{\cos (\alpha+\varphi)+\sqrt{2} \alpha \cdot \sin \left(\alpha+\varphi+\frac{\pi}{4}\right)} \int_{0}^{\infty} e^{-\alpha y}\left[\frac{\sin (\alpha(1+y)+\varphi)}{(1+y)^{2}},\right. \\
& \left.-\frac{\sqrt{2} \cdot \alpha}{1+y} \cos \left(\alpha(1+y)+\varphi+\frac{\pi}{4}\right)\right] d y
\end{aligned}
$$

Let's now make following substitutions, $\alpha(1+y)=t$ and $\alpha d y=d t$.

We then have:

$$
\begin{aligned}
\operatorname{Re}(u)= & \frac{E_{20} \cdot \alpha e^{\alpha}}{\cos (\alpha+\varphi)+\sqrt{2} \alpha \cdot \sin \left(\alpha+\varphi+\frac{\pi}{4}\right)} \int_{\alpha}^{\infty} \frac{e^{-t}}{t^{2}}[\cos (t+\varphi), \\
& +t(\sin (t+\varphi)+\cos (t+\varphi))] d t \\
\operatorname{Im}(u)= & \frac{E_{20} \cdot \alpha e^{\alpha}}{\cos (\alpha+\varphi)+\sqrt{2} \alpha \cdot \sin \left(\alpha+\varphi+\frac{\pi}{4}\right)} \int_{\alpha}^{\infty} \frac{e^{-t}}{t^{2}}[\sin (t+\varphi), \\
& +t(\sin (t+\varphi)-\cos (t+\varphi))] d t
\end{aligned}
$$

Integrating by parts, we obtain the following: 


$$
\begin{aligned}
& \operatorname{Re}(u)=\frac{E_{20} \cdot \cos (\alpha+\varphi)}{\cos (\alpha+\varphi)+\sqrt{2} \alpha \cdot \sin \left(\alpha+\varphi+\frac{\pi}{4}\right)} \\
& \operatorname{Im}(u)=\frac{E_{20} \cdot \sin (\alpha+\varphi)}{\cos (\alpha+\varphi)+\sqrt{2} \alpha \cdot \sin \left(\alpha+\varphi+\frac{\pi}{4}\right)}
\end{aligned}
$$

Simplifies to:

$$
\begin{aligned}
\operatorname{Re}(u) & =\frac{\text { const } \cdot i}{1+\alpha \cdot[\operatorname{tg}(\alpha+\varphi)+1]} \\
\operatorname{Im}(u) & =\frac{\text { const } \cdot i \cdot \operatorname{tg}(\alpha+\varphi)}{1+\alpha \cdot[\operatorname{tg}(\alpha+\varphi)+1]}
\end{aligned}
$$

We now have an expression for the complex impedance $Z$ as:

$$
\begin{aligned}
& \operatorname{Re}(Z)=\frac{R_{0}}{1+\alpha \cdot[\operatorname{tg}(\alpha+\varphi)+1]} \\
& \operatorname{Im}(Z)=\frac{R_{0} \cdot \operatorname{tg}(\alpha+\varphi)}{1+\alpha \cdot[\operatorname{tg}(\alpha+\varphi)+1]}
\end{aligned}
$$

where $R_{0}$ is the Butler/Volmer DC resistance of the charge transfer reaction, according to equation 44 .

By inserting $\varphi$ from equation 106 we will finally, after an additional few steps of reduction and simplification, arrive at the following expression for the complex impedance $Z$ :

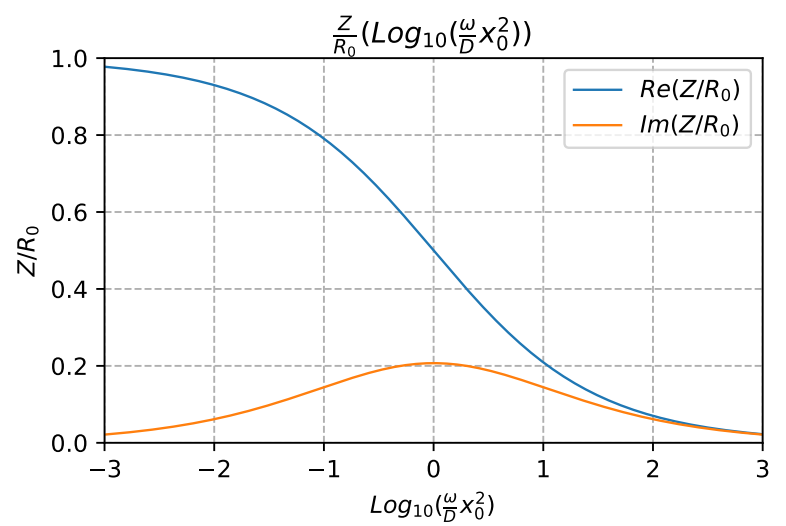

Figure 14: The Complex Impedance

$$
\operatorname{Re}(Z)=R_{0} \frac{\alpha+1}{(\alpha+1)^{2}+\alpha^{2}}
$$




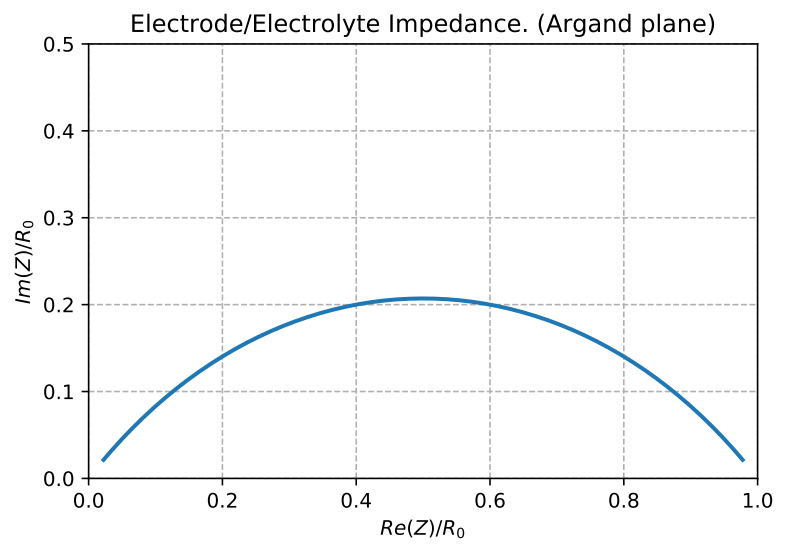

Figure 15: The Complex Impedance - Argand plane

$$
\operatorname{Im}(Z)=R_{0} \frac{\alpha}{(\alpha+1)^{2}+\alpha^{2}}
$$

Amplitude maximum of equation 122 occurs at $\alpha=\frac{1}{\sqrt{2}}$. From this follows:

$$
\alpha=\sqrt{\frac{\omega}{2 \omega_{0}}}
$$

where $\omega_{0}$ is the frequency at $\operatorname{Im}(Z)$ maximum.

Figure 14 shows the normalized complex impedance $Z / R_{0}$ as a function of normalized frequency $\frac{\omega}{D} x_{0}^{2}$, while figure 15 shows $Z / R_{0}$ in the Argand plane.

\subsubsection{Experimental Results}

We will in this section compare the theoretically derived complex impedance with experimental data obtained using an acidic $\mathrm{Cu} / \mathrm{CuSO}$ half-cell (Nilsson and Bockris, 1976).

Three different concentrations of $\mathrm{CuSO}_{4}$ were used, 1.0, 0.1, and $0.01 \mathrm{~mol} / \mathrm{L}$. Each mixed with $\mathrm{H}_{2} \mathrm{SO}_{4}$ at three different concentrations, $1.0,0.1$, and $0.01 \mathrm{~mol} / \mathrm{L}$. Thus a total of nine different electrolyte mixtures were used.

Figure 16 shows the experiment setup, with the purification vessel near the center of the photo. We discern the white half-cell made out of Teflon to its left and slightly below.

The cylinder visible on the left provided the nitrogen used for the de-aeration of the electrolyte.

The complex impedance data was measured using an instrument developed specifically for the purpose by Boliden and designated Z-Lab-71 (Nilsson and Wikman, 1971). 


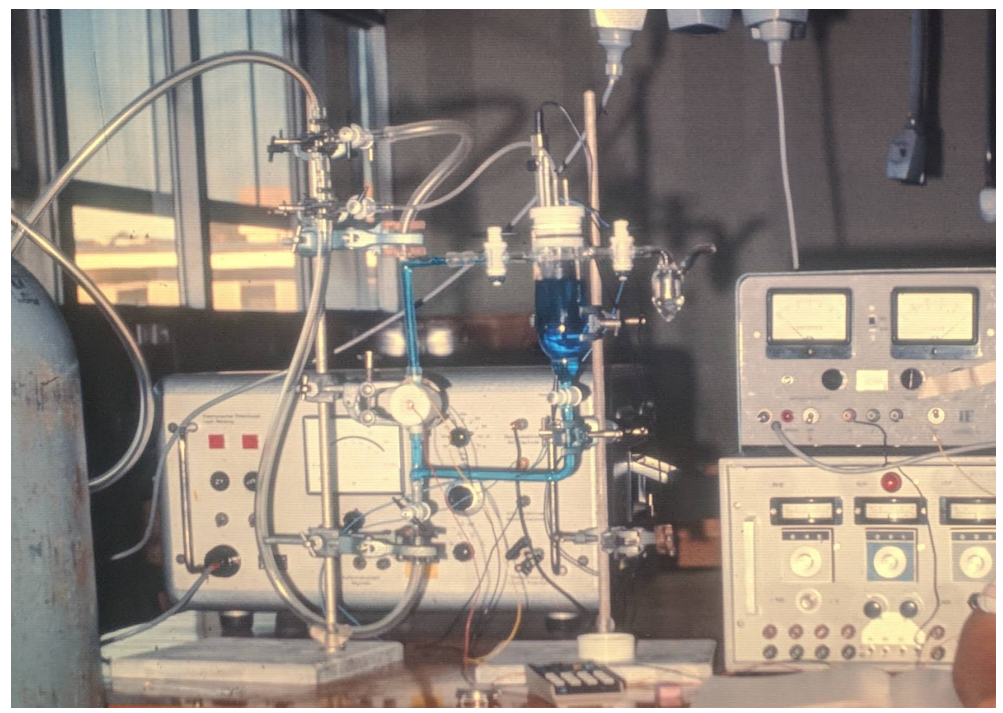

Figure 16: Experiment Setup

The instrument, visible in the lower right of the photo, was designed to measure the real and the imaginary components of the cell impedance over a range of logarithmically spaced frequencies from $0.07 \mathrm{~Hz}$ to $2400 \mathrm{~Hz}$.

We utilized low currents, $50 \mu \mathrm{A}$ or $150 \mu \mathrm{A}$, to ensure the electrode voltage would remain well within the cell's linear range, typically within $\pm 1 \mathrm{mV}$ of its equilibrium voltage.

The output impedance of the instrument's current output stage was a high $50 M \Omega$ to keep the current unaffected of the cell impedance.

The measurement accuracy was better than $0.5 \%$ for both components. We regularly monitored the absolute accuracy of the instrument by measuring the impedance of a parallel precision combination of a $100 \Omega$ resistor and a $40 \mu \mathrm{F}$ capacitor. Figure 17 shows the result of one such measurement, including the best fit with the characteristic impedance for such a network.

It is important here to point out that the impedance for the electrode/electrolyte interface, as shown in figure 14, is quite different in character and cannot be realized with a regular resistor/capacitor network since the components would have to be frequency-dependent.

A total of 151 measurements were carried out. Figure 18 shows typical results for three different $\mathrm{CuSO}_{4}$ concentrations.

The electrolyte was pre-electrolyzed nightly via a platinum electrode held at $0.7 \mathrm{~V}$ and kept de-aerated by bubbling nitrogen gas through the cell.

The planar $\mathrm{Cu}$-electrode had a $1 \mathrm{~cm}$ diameter circular active area. A new electrode was slightly etched in $\mathrm{HNO}_{3}$, washed in $\mathrm{H}_{2} \mathrm{SO}_{4}$, and then cleaned in distilled water before being inserted into the cell.

Before each measurement, the electrode was plated using a two mA current 


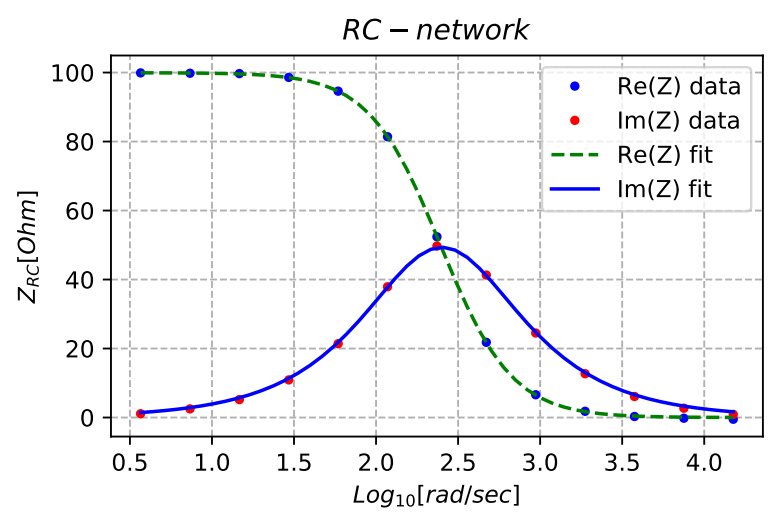

Figure 17: Instrument calibration test

for 30 minutes and then after a 10 min waiting period, the impedance measurement would commence.

If we look at the leftmost plot in figure 18, we recognize the general shape of the theoretical impedance function.

However, when we examine the rightmost plot, we notice deviations at low and high frequencies. The measurements, in this case, were done at the lowest $\mathrm{CuSO}_{4}$ concentration. Both the imaginary and the real component amplitudes increase at the low-frequency end as the frequency decreases due to the diffusion over-voltage. At the high-frequency end, it is evident that the real component appears to be shifted by a constant amount. The increased resistance of the electrolyte and the fact that the reference electrode was $5 \mathrm{~mm}$ away from the electrode surface explain this. Since the electrolyte resistance, $R_{e}$ is in phase with the measurement current; it only shows up in the real component of the impedance.

The Warburg impedance $Z_{w}$ describes the diffusion over-voltage well:

$$
Z_{w}=R_{w} \frac{1}{\sqrt{j \omega}}
$$

where $R_{w}$ is a constant.

The total impedance, using equations 123, and 124, can thus be formulated, as:

$$
\left\{\begin{array}{l}
\operatorname{Re}\left(Z_{t o t}\right)=R_{0} \frac{\alpha+1}{(\alpha+1)^{2}+\alpha^{2}}+R_{w} \frac{1}{\sqrt{\omega}}+R_{e} \\
\operatorname{Im}\left(Z_{t o t}\right)=R_{0} \frac{\alpha}{(\alpha+1)^{2}+\alpha^{2}}+R_{w} \frac{1}{\sqrt{\omega}} \\
\alpha=\sqrt{\frac{\omega}{2 \omega_{0}}}
\end{array}\right.
$$

The Python plotting program used to plot the graphs in figure 18 was combined with a curve fitting function, based on the Levenberg-Marquardt algo- 
rithm, to extract the unknown parameters, $R_{0}, R_{w}, R_{e}, \omega_{0}$ and their standard deviations, from equations 125 .

The width of the electric field distribution, as indicated by the parameter $x_{0}$, was calculated via equations 107 and 123 as: $x_{0}=\sqrt{\frac{D}{\omega_{0}}}$ where $D=6 \cdot 10^{-10}$.

The exchange current $i_{0}$ was likewise obtained by utilizing equation 44 and the $R_{0}$ parameter.

As is evident from the excellent fit, as indicated in figure 18, the measurements are very well described by the impedance function $Z_{t o t}$.
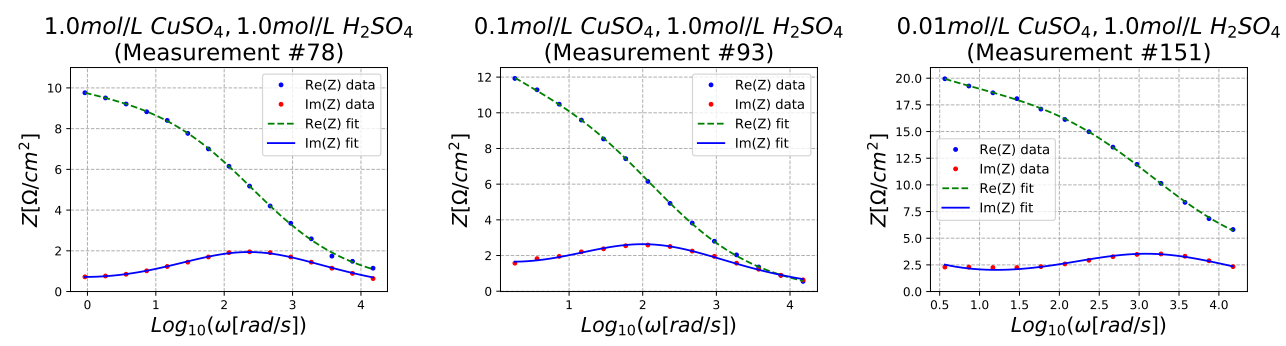

Figure 18: Complex Impedance. Measurements \#78, \#93 and \#151

Table 1 shows the mean and standard deviation of the extracted parameters for the same measurement samples.

\begin{tabular}{|l|l|l|l|}
\hline & $\# 78$ & $\# 93$ & $\# 151$ \\
\hline$R_{0}\left[\Omega \mathrm{cm}^{2}\right]$ & $9.5 \pm 0.1$ & $12.1 \pm 0.2$ & $16.8 \pm 0.3$ \\
\hline$R_{w}\left[\Omega \sqrt{\frac{r a d}{s e c}} \mathrm{~cm}^{2}\right]$ & $0.3 \pm 0.1$ & $1.3 \pm 0.2$ & $3.2 \pm 0.4$ \\
\hline$R_{e}\left[\Omega \mathrm{cm}^{2}\right]$ & $0.2 \pm 0.1$ & $0.0 \pm 0.2$ & $2.2 \pm 0.2$ \\
\hline$\omega_{0}[\mathrm{rad} / \mathrm{sec}]$ & $247 \pm 17$ & $126 \pm 8$ & $1415 \pm 142$ \\
\hline$x_{0}[\mu \mathrm{m}]$ & $1.56 \pm 0.05$ & $2.18 \pm 0.07$ & $0.65 \pm 0.03$ \\
\hline$i_{0}\left[\mathrm{~mA} / \mathrm{cm}^{2}\right]$ & $1.37 \pm 0.02$ & $1.07 \pm 0.01$ & $0.77 \pm 0.01$ \\
\hline \hline
\end{tabular}

Table 1: Extracted parameters. (Measurements \#78, \#93 and \#151

Even though the measurement accuracy was quite good for individual measurements, superimposed was a longer-term drift that significantly affected the accuracy over time.

Thus, when averaging repeated measurements, the standard deviations of the extracted parameters illustrate the extent of this issue.

The reason for this variability is the difficulty in maintaining stable and well-defined conditions at the electrode surface.

Figure 19 shows the relation between the $x_{0}$ and $i_{0}$ parameters with electrolyte activity. It shows the mean of all measurements done for each of the nine electrolytes. 
The activity for each electrolyte was calculated from the equilibrium electrode voltage, as measured with a Calomel reference electrode, using Nernst's equation.

The red lines show the linear regression results.

With the $x_{0}$ parameter virtually independent of activity we obtained as an average $x_{0}=1.0 \mu \mathrm{m} \pm 16 \%$.

For $i_{0}$ we obtained $i_{0}=2.5 \cdot a^{0.4} \mathrm{~mA} / \mathrm{cm}^{2} \pm 13 \%$. The relationship with activity is reasonably close to the predicted square root relationship, as is indicated by the green line, considering the variability of the data.
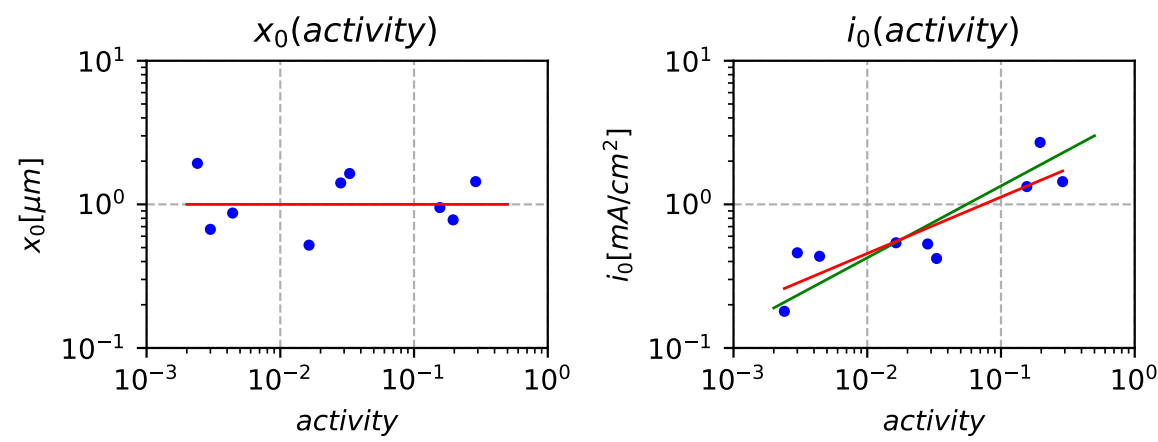

Figure 19: Electric Field thickness and Exchange Current as function of activity

Many teams have measured the exchange current of $\mathrm{Cu} / \mathrm{CuSO} \mathrm{S}_{4}$ cells over the last 70 years. The spread between results covering a range of almost two orders of magnitude illustrates both a problem with the cell itself and the measurement techniques. (Hurlen, 1961) provided a good review of the early results.

The advantage of the present experimental technique is that we benefit from an accurate model of the complex impedance, which minimizes model-related errors. Another advantage is that the measurements were done right at the equilibrium voltage with a small $\mathrm{AC}$ voltage amplitude of less than $1 \mathrm{mV}$.

\section{Computer Programs}

The computer programs used to provide the numerical solutions to the differential equations, and to generate the various figures, were all written in the Python language. We have made them publicly available in the form of a Python Notebook that resides at:

https ://github.com/byn4201/El_Chem

and is called: A_General_Theory.ipynb

One way to run the Notebook, and the individual programs, is to use Google's Colaboratory, which runs from the browser. The user can play with parameters and change the code or the graphics output without interfering with the underlying code. 
(https://colab.research.google.com/notebooks/welcome.ipynb)

\section{Conclusions}

The presented theoretical model provides a detailed and accurate description of the electric field and the ion distribution near an electrode, both in the space and time domain.

This in turn, provides a new and fundamental foundation for describing both static and dynamic properties of the electrode/electrolyte interface.

To illustrate the general validity of the theory, we first derived the well-known Butler/Volmer and Nernst's Equations.

Of particular utility is the theory's ability to deal with dynamic properties, like the variation of the electrode voltage from the moment an electrode makes contact with an inert electrolyte.

We carried out experiments whereby a $Z n$ electrode was dipped into a $\mathrm{Na}_{2} \mathrm{SO}_{4}$ electrolyte. The recorded electrode voltage variation with time showed excellent agreement with the theory.

We also used the theory to derive the complex impedance of the electrode/electrolyte interface. We compared the theory with experimental data, in this case with impedance measurements on a $\mathrm{Cu} \mid \mathrm{CuSO} \mathrm{S}_{4} / \mathrm{H}_{2} \mathrm{SO}_{4}$ cell.

Individual impedance measurements showed an excellent fit with theory, allowing us to determine the exchange current $i_{0}$ with an accuracy of better than $2 \%$. Likewise, the electric field distribution thickness parameter $x_{0}$ was obtained with an accuracy of better than $3 \%$.

\section{Acknowledgments}

The support provided by Boliden Mining, Boliden, Sweden, has made this project possible.

The National Swedish Board for Technical Development provided financial support for the experimental study of the complex impedance of the electrode/electrolyte interface at Flinders University of South Australia, Adelaide.

Professor John O'M Bockris, Flinders University, provided valuable advice and made his laboratory and support staff available for the experiments.

His two-volume book-set, Modern Electrochemistry, provided inspiration and was the 'bible' during this 'on-off-on' fifty-two year (!) project.

\section{References}

Bauer, H. H., 1968, The electrochemical transfer-coefficient: Journal Electroanalytical Chemistry and Interfacial El.Chem., , no. 16, 419-432.

Bockris, J. O., and Reddy, A. K. N., 1970, Modern electrochemistry: Plenum Press. 
Chapman, D. L., 1913 Phil. Mag., 25, no. 6, 475.

Gouy, G., 1903 J. Chim. Phys., 29, no. 7, 145.

Hurlen, T., 1961, On th kinetics of the $\mathrm{cu} / \mathrm{cu}++$ aq electrode: Acta Chemica Scandinavica, , no. 15 .

Nilsson, B., and Bockris, J. O., Lab notebook 1 and 2, cu/cu++aq impedance measurements:, 1976.

Nilsson, B., and Wikman, L., Z-lab-71. design and development:, 1971.

Nilsson, B., 1970, A new combined resistivity and induced polarization instrument and a new theory of the induced polarization phenomenon: Geoexploration, 1971, no. 9, 35-54.

Nilsson, B., A theory of electrical phenomena at an electrode/electrolyte interface (in swedish):, Technical report, Boliden Mining, Sweden, 1973.

Nilsson, B., The complex impedance of an electrode/electrolyte system. (in swedish):, Technical report, Boliden Mining, Sweden, 1975.

Stern, O., 1924 Z. Electochem., , no. 30, 508.

von Helmholtz, H., 1879, Studien ueber electrische grenzschichten: Annalen der Physik und Chemie, 7, 337. 